\title{
Multimedia Transmission through Mobile Ad hoc Networks using Multicasting
}

\author{
Lavanya. Poluboyina, Sivakumar Reddy. V, Mallikarjuna Prasad. A
}

\begin{abstract}
Mobile ad hoc networks, abbreviated to MANETs are covering various application fields with the significant advancements in wireless networks. In the recent past, the multimedia transmission over such networks has drawn the attention from researchers and scientists. In wireless medium, it is always advantageous to go for multicast routing than unicast routing. This paper focuses on the performance analysis of a multicast routing protocol, multicast ad hoc on-demand distance vector (MAODV), and its modified version QoS-MAODV, to support multimedia transmission over ad hoc environments.
\end{abstract}

Keywords: MANET, MAODV, mobile ad hoc network, multimedia, VoIP transmission, video transmission.

\section{INTRODUCTION}

Out of the two diverse approaches for enabling wireless communication between two mobile users, the mobile users forming a temporary network instantly, called an ad hoc network has got its attention especially for certain applications like battlefield, emergency situations, disasters, natural calamities etc. The mobile ad hoc network (MANET) contains autonomously connected mobile nodes without any centralized administration/networking infrastructure. This network has a shortcoming: the network is limited to the individual node's transmission range and hence depends on the nodes to give consent for forwarding the data packets to distant destinations that are not in direct communication range. The communication between the source and the destination may be a single hop or may take multiple hops depending on the positions of them.

MANETs have to provide services in hostile environments where establishing a robust path is difficult, in addition to the fact that the nodes in these networks are mobile in nature, leading to dynamic network topologies. Multi-hop networking, mobility, wireless channel combined with limited bandwidth, device heterogeneity, battery power limitations are the factors causing the routing protocols design a complex problem in MANETs.

Revised Manuscript Received on February 05, 2020.

* Correspondence Author

Lavanya. Poluboyina*, Department of ECE, Sreenidhi Institute of Science and Technology, Hyderabad, Telangana, India. Email: lavanyamam@yahoo.com

Sivakumar Reddy. V, Department of ECE, Mallareddy College of Engineering \& Technology, Hyderabad, Telangana, India. Email: vskreddy2003@gmail.com

Mallikarjuna Prasad. A, Department of ECE, JNTUK, Kakinada, Andhra Pradesh, India. Email: a_malli65@yahoo.com

(c) The Authors. Published by Blue Eyes Intelligence Engineering and Sciences Publication (BEIESP). This is an open access article under the CC BY-NC-ND license (http://creativecommons.org/licenses/by-nc-nd/4.0/)
Two classes of unicast routing protocols based on their route finding strategy are: table-driven type which are reliable and maintain up-to-date routing information in the form of routing tables. Whereas the on-demand type protocols find routes only when it is required that results in less control overhead in the network. Either the source or destination node initiates the route finding process if that required route information is not available in their routing table. Henceforth, it is obvious that there is no guarantee of a route in the case of on-demand routing protocols unlike table-driven. Multicasting is the group communications where the data packets are transmitted from a source node (must be a group member) to the remaining group members with one click. The nodes are said to be group members if they possess the same single multicast group address [1]. Due to its low routing overhead requirement, multicasting in MANETs supports diverse applications such as collaborative computing etc. One category of multicast routing protocols, based on multicast group forming strategy, is tree-based [2]. The multicast extension of AODV (ad hoc on-demand distance vector) is the MAODV (Multicast Ad hoc On-demand Distance Vector) which is basically a shared-tree based. In this paper, to have improved performance to support multimedia, some modifications have been done to the basic operation of MAODV, referred to as QoS-MAODV, and the performance of both the protocols is compared for a set of quality of service (QoS) support parameters. The presentation is organized in the following way: in sections II and III, the MAODV and QoS-MAODV are discussed in brief respectively. Section IV provides the information about the work environment. The obtained results are analyzed in section V. Conclusions are marked in section VI.

\section{MAODV}

Follows on-demand strategy implies routes are discovered only whenever needed. Each node holds three tables: routing table, request table and multicast routing table [3]. Routing table functions same as the routing table of AODV, carries routing information. Request table records the route requesting node's IP addresses and the requested multicast group IP address. Multicast routing table contains multicast groups related information like sequence number, IP address of group leader, group address, next hops, and hop count to group leader and member. Node that wants to be part of the multicast group, broadcasts the route request packet when it has no route to that group. On receiving a route request message, a member node of the desired group responds whereas a non-member rebroadcasts the request and stores the necessary routing information for reverse routing paths. 
Any node with fresh route to multicast group responds to route request message with non-join route request message. Non-join route requests are handled in the way same as AODV. An additional entry is being added to the multicast routing table for join route request messages and this entry will be activated only when the node is selected to be part of the multicast distribution tree.

The desired multicast group's member replies to the route request messages only if the recorded sequence number to that group is greater than the one contained in the route request message. The responding node generates and unicasts a route reply message back to the route requested node after updating relevant fields of its routing table and multicast routing tables. Both the routing and multicast routing table entries of the nodes along the path to the source node are also updated to create the forward path.

Hello messages are broadcasted periodically to detect the broken links. By setting TTL value to one in hello messages, local connectivity is maintained. When a node broadcasting hello messages is not heard from another node for several allowed attempts, the link is assumed to be off between the two nodes, and the expanding ring search algorithm is used for connection repair.

\section{QOS-MAODV}

There have been various methodologies proposed for providing QoS using multicasting [4-5]. QoS-MAODV is the modified version of MAODV. Route discovery phase uses the route request and route reply messages. The extensions have been added to these messages to provide QoS. Admission control is implemented to prevent intermediate nodes from being overload. When an intermediate node receives a QoS-route request packet, and has enough available bandwidth, it accepts the route request. The intermediate node rejects the route request packets of new sessions, if there is no available bandwidth.

The additional message used is QoS in QoS-MAODV. This message informs the other nodes about the reserved bandwidth. The format of all the messages remains as specified in [4] except some flags and extensions. These flags and extensions are added for supporting state of bandwidth reservation. The nodes update their state of bandwidth reservation based on hello and group hello messages received at certain times.

It has the additional tables: 1 ) bandwidth reservation table used to keep bandwidth reservation information for different groups. The entries in this table are multicast group address, amount of reserved bandwidth, state of the reservation, time stamp, hop count from the source node, and source node's IP address, 2) neighbors table keeps the information such as neighbor address, amount of reserved bandwidth in neighbor node, state of the reservation, amount of consumed bandwidth in neighbor, state of neighbor (sender, receiver or forwarding node), and time stamp, and 3) multicast consumed bandwidth keeps required information for calculation of consumed bandwidth in each node. Reserved bandwidth for uplink and downlink nodes in multicast tree and the state of reservation are kept in this table.

Whenever a member of the multicast group wishes to initiate a transmission, it broadcasts a route request message with the bandwidth field set to required bandwidth. On receiving a route request message a multicast member/forwarding node responds with a route reply message only when the required amount of bandwidth is available. The nodes that reserve (at this stage, it is just a temporary) the bandwidth on accepting the request, in fact, update their tables by recording the sequence numbers and next hop address to the multicast source. The multicast source computes the end to end delay for the received single or multiple (if receives) route reply messages, and selects the path that has minimum end to end delay and hop count to the multicast group leader. In addition, it sends the path activation message to change the state of bandwidth to reserve. For a certain interval of time, if the multicast source not received any route reply messages, then it rebroadcasts the route request message and there is a limit on the number of retries. Along the established path, if any node detects that it no longer can reserve the required bandwidth then it sends that message back to the node which had requested for the reservation of resource.

\section{WORK ENVIRONMENT}

A MANET of 50 mobile nodes moving with a maximum speed of $2.5 \mathrm{~m} / \mathrm{s}$ is considered in the simulation area of $1000 \mathrm{mx} 1000 \mathrm{~m}$. The simulation is done with the open network simulator tool, ns 2.34 version [6]. The in-built functions are utilized for the generation of traffic files and for the mobility of the nodes (random waypoint mobility. The G.711 codec and the Evalvid tool-set are used for the generation of VoIP and video traffic respectively [5]. Both the node mobility and the network are simulated for 600s. IEEE 802.11 MAC with two-ray ground propagation model is taken for simulation. A data packet rate of 4 with CBR and a packet size of 512 bytes is identified for the cases of CBR and video traffics.

First, the performance of both MAODV and QoS-MAODV are examined for 1, 2, and 3 multicast groups and for the three traffic types (CBR, VoIP, and video) separately. Each multicast group of size 10 nodes and at any instant of time only one multicast sender will be there. In second experiment, 2 different multimedia traffic files are created for the verification of working of MAODV and QoS-MAODV for multimedia traffic. In MM traffic 1 file, 3 multicast groups and each for one type of traffic are defined whereas in MM traffic 2 file, one multicast group with three sources identified for three different traffic types at different times. The average of results obtained for 10 different scenario files are plotted for the parameters packet received successfully (packet delivery ratio-PDR), average delay, delay variation and normalized routing overhead (NRO).

\section{RESULTS AND DISCUSSIONS}

The simulated results are tabulated in the three tables, Table I is showing MAODV results for 1, 2, and 3 multicast groups and for the three traffic types. Similarly, Table II is for QoS-MAODV and the Table III providing combined results for two different multimedia traffic files.

Fig.s 1 to 4 are showing the plots for PDR in \%, average delay in ms, delay variation in $\mathrm{ms}$, and $\mathrm{NRO}$ in \% for MAODV and QoS-MAODV for CBR traffic.

Efficient routing protocol implies large PDR values and, low values of delay and routing overheads. 
It is evident from all figures that the performance of the QoS-MAODV is superior to MAODV. Moreover, it is obvious that PDR values decreases with increasing number multicast groups and the reverse case to delay values. The overloading of queues at forwarding nodes with increase in number of traffic connections has led to the packet drops in these low-mobility network environments. From the NRO plots, it is noted that with increased number of multicast groups the routing overhead has been decreased.

Table- I: Results of MAODV for three different traffic types

\begin{tabular}{|l|c|c|c|}
\hline \multirow{2}{*}{$\begin{array}{c}\text { Performance } \\
\text { Metric }\end{array}$} & 1MG Number of Multicast Groups \\
\cline { 2 - 4 } & \multicolumn{3}{|c|}{ CBR } \\
\hline Ratio (\%) & 94.2773623 & 84.8001752 & 75.4195141 \\
\hline Latency (ms) & 46.5248366 & 105.848864 & 355.901283 \\
\hline Jitter (ms) & 49.7119943 & 96.2948674 & 268.214529 \\
\hline $\begin{array}{l}\text { Normalized } \\
\text { Routing Load (\%) }\end{array}$ & 35.3661997 & 16.1048869 & 11.3684826 \\
\hline \multicolumn{4}{|c|}{ VoIP } \\
\hline Ratio (\%) & 68.2470558 & 46.0133639 & 38.2224132 \\
\hline Latency (ms) & 809.942735 & 1626.93912 & 1943.3135 \\
\hline Jitter (ms) & 597.686099 & 1184.63011 & 1592.06225 \\
\hline $\begin{array}{l}\text { Normalized } \\
\text { Routing Load (\%) }\end{array}$ & 7.32512479 & 3.20430219 & 4.3390965 \\
\hline \multicolumn{4}{|c|}{ Video } \\
\hline Ratio (\%) & 32.7121097 & 26.442625 & 23.7907826 \\
\hline Latency (ms) & 1832.67581 & 2205.83291 & 2514.83669 \\
\hline $\begin{array}{l}\text { Jitter (ms) } \\
\text { Normalized } \\
\text { Routing Load (\%) }\end{array}$ & 358.442501 & 168.20981 & 88.4883713 \\
\hline
\end{tabular}

Table- II: Results of QoS-MAODV for three different traffic types

\begin{tabular}{|l|c|c|c|}
\hline \multirow{2}{*}{$\begin{array}{c}\text { Performance } \\
\text { Metric }\end{array}$} & 1MG Number of Multicast Groups \\
\cline { 2 - 4 } & \multicolumn{3}{|c|}{ CBR } \\
\hline Ratio (\%) & 98.6435692 & 95.0136261 & 84.716821 \\
\hline Latency (ms) & 11.3789268 & 53.259531 & 114.494313 \\
\hline Jitter (ms) & 6.5843186 & 25.3843369 & 54.6304633 \\
\hline $\begin{array}{l}\text { Normalized } \\
\text { Routing Load (\%) }\end{array}$ & 25.5571546 & 10.6997285 & 5.7498656 \\
\hline \multicolumn{4}{|c|}{ VoIP } \\
\hline Ratio (\%) & 81.5192578 & 56.8922714 & 40.9680604 \\
\hline Latency (ms) & 200.885953 & 683.81971 & 1106.62576 \\
\hline Jitter (ms) & 85.5660475 & 227.034359 & 631.454757 \\
\hline $\begin{array}{l}\text { Normalized } \\
\text { Routing Load (\%) }\end{array}$ & 3.63052337 & 1.87620849 & 2.20893611 \\
\hline \multicolumn{4}{|c|}{ Video } \\
\hline Ratio (\%) & 45.5392232 & 41.4767708 & 28.7846568 \\
\hline Latency (ms) & 790.251092 & 998.644866 & 1448.01746 \\
\hline Jitter (ms) & 319.377293 & 365.358012 & 554.304567 \\
\hline $\begin{array}{l}\text { Normalized } \\
\text { Routing Load (\%) }\end{array}$ & 175.087655 & 67.7319717 & 63.8941884 \\
\hline
\end{tabular}

Table- III: Results of MAODV and QoS-MAODV for two different multimedia traffic files

\begin{tabular}{|l|l|l|l|l|}
\hline $\begin{array}{c}\text { Performance } \\
\text { Metric }\end{array}$ & \multicolumn{2}{|c|}{ MAODV } & \multicolumn{2}{c|}{ QoS-MAODV } \\
\hline & $\begin{array}{c}\text { MM } \\
\text { Traffic 1 }\end{array}$ & $\begin{array}{c}\text { MM } \\
\text { Traffic 2 }\end{array}$ & $\begin{array}{c}\text { MM } \\
\text { Traffic 1 }\end{array}$ & $\begin{array}{c}\text { MM } \\
\text { Traffic 2 }\end{array}$ \\
\hline Ratio (\%) & 51.17 & 53.77 & 38.91 & 80.04 \\
\hline Latency (ms) & 1499.18 & 682.99 & 1282.00 & 125.71 \\
\hline
\end{tabular}

\begin{tabular}{|l|l|l|l|l|}
\hline Jitter (ms) & 904.26 & 493.61 & 244.33 & 52.81 \\
\hline $\begin{array}{l}\text { Normalized } \\
\text { Routing Load } \\
\text { (\%) }\end{array}$ & 4.65 & 5.00 & 4.43 & 2.71 \\
\hline
\end{tabular}

PDR in \%, average delay in ms, delay variation in ms, and NRO in \% for MAODV and QoS-MAODV for VoIP traffic, are shown in the fig.s 5 to 8 .

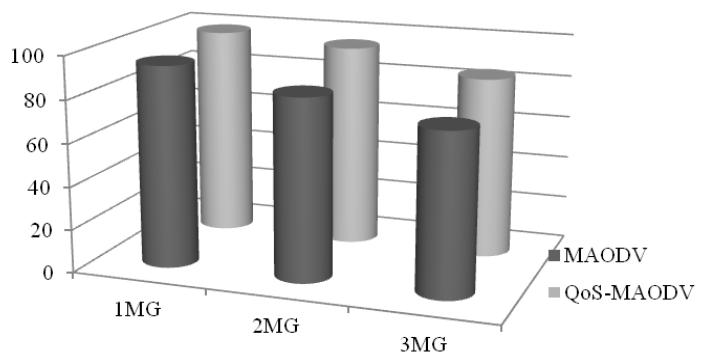

Fig. 1.CBR-PDR.

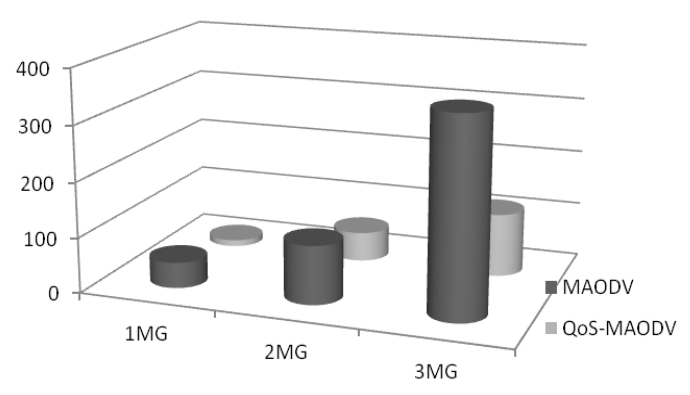

Fig. 2.CBR-Average delay

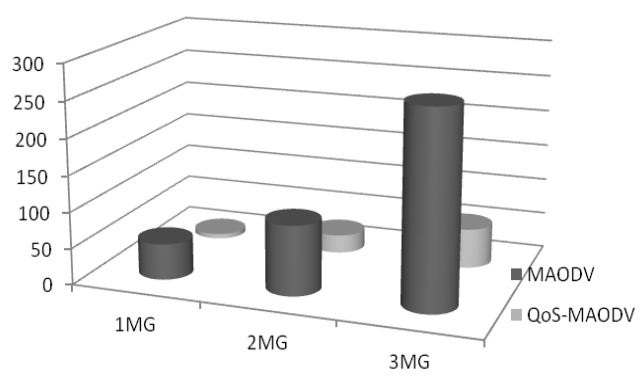

Fig. 3.CBR-Delay variation.

From the MAODV's VoIP and video plots, it is clearly noted that the basic MAODV is not suitable for multimedia transmission due to its low PDR and high delay values. Moreover, very low PDR and very high delay values are identified with video traffic compared to VoIP traffic cause of high video packet transmission rates.

Though improved PDR and delay values are obtained with modified MAODV, these values are not sufficient to provide the acceptable QoS support for multimedia. From the NRO plots of both VoIP and video traffic, it is noted that the amount of routing overhead required for VoIP traffic is very much less than the video case.

Published By:

Blue Eyes Intelligence Engineering

\& Sciences Publication

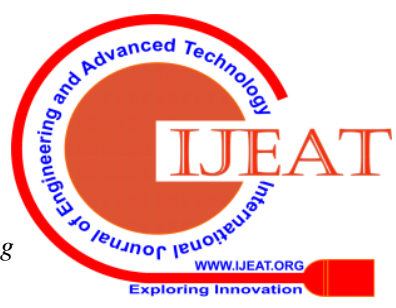




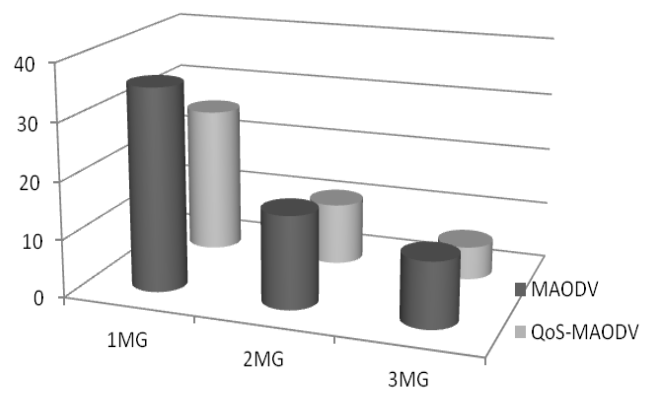

Fig. 4.CBR-NRO.

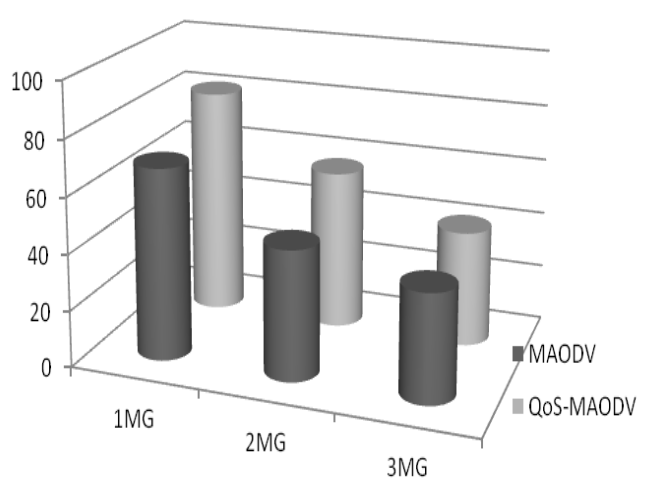

Fig. 5.VoIP-PDR.

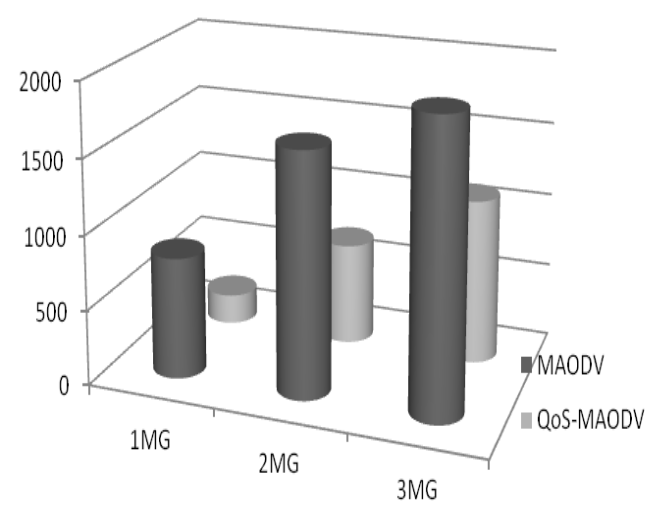

Fig. 6.VoIP-Average delay.

From the multimedia plots, neither PDR values nor the delay values are adequate enough to accommodate multimedia services. The benefit of multicast routing is self-explanatory with the obtained routing overhead values.

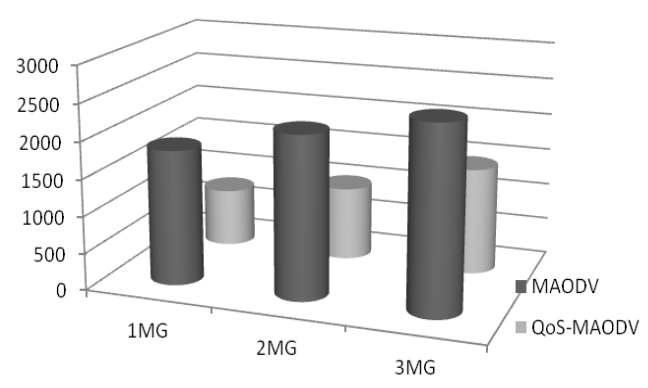

Fig. 10. Video-Average delay.

Fig. 9.Video-PDR.

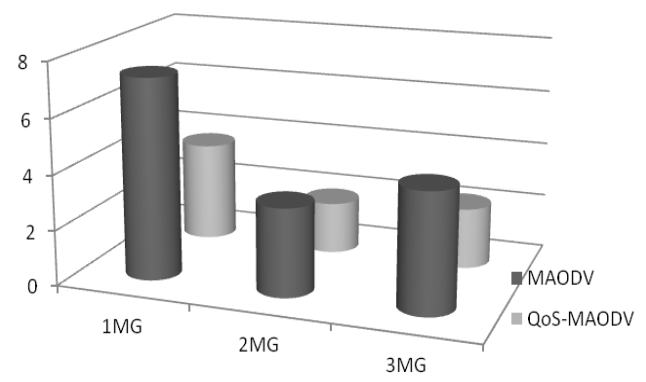

Fig. 8.VoIP-NRO.

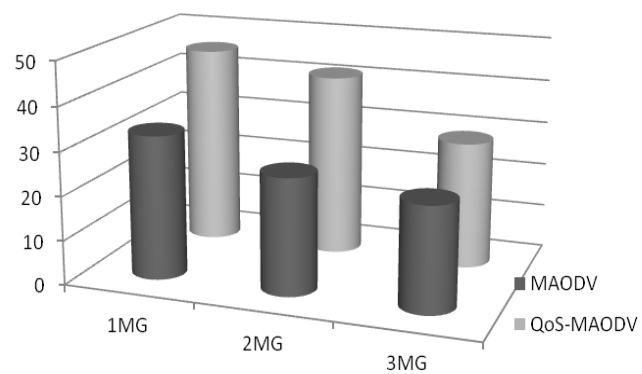




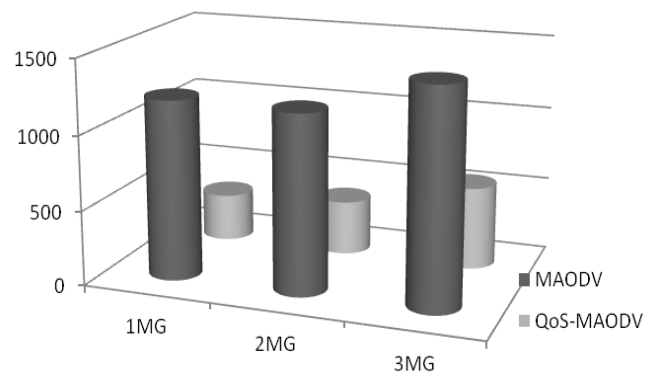

Fig. 11. Video-Delay variation.

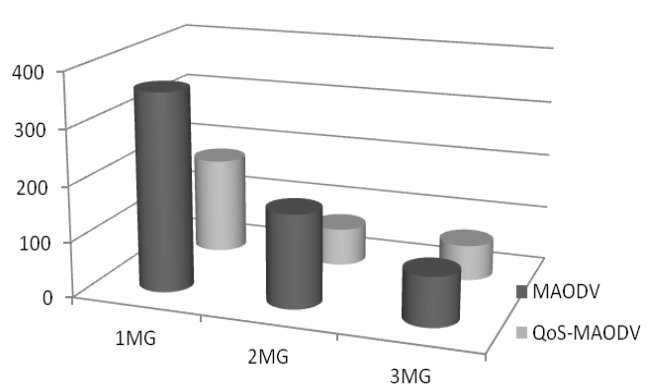

Fig. 12. Video-NRO.

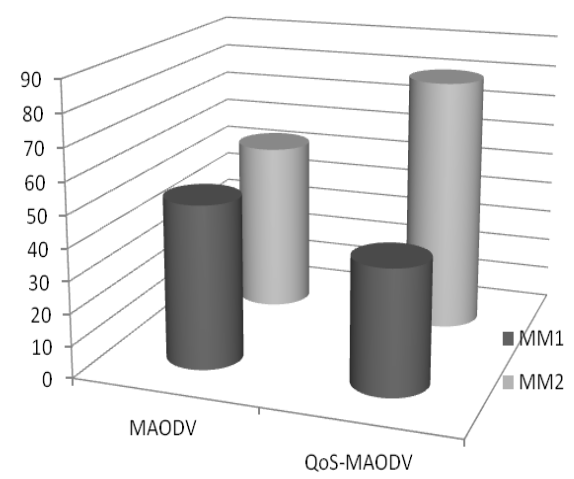

Fig. 13. Multmedia-PDR.

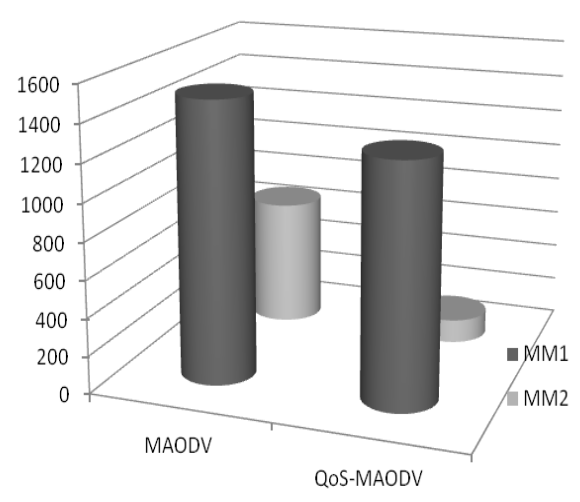

Fig. 14. Multmedia-Average delay.

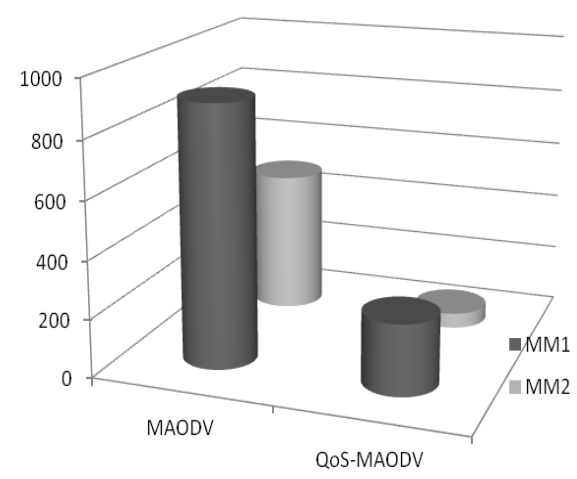

Fig. 15. Multmedia-Delay variation.

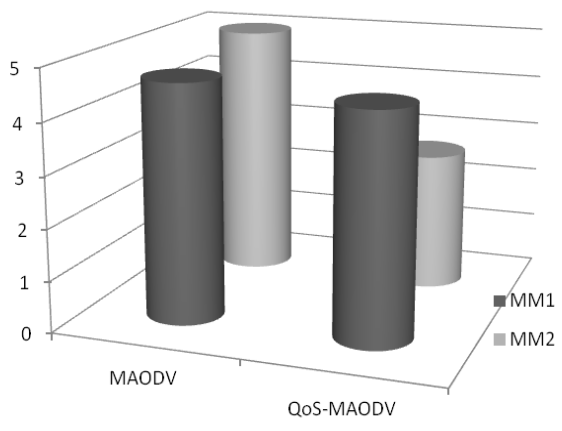

Fig. 16. Multmedia-NRO.

\section{CONCLUSION}

The demand for multimedia transmission over ad hoc environments to support various applications and to exploit the multicast routing to reduce the amount routing overhead required, the basic MAODV's and its modified version QoS-MAODV's performance are assessed for different types of data traffics, and for multimedia.

From the obtained results it is clearly observed that the work done is not sufficient and further investigations to the behavior of multicast routing is required for further improving the QoS parameters to support multimedia transmission over MANETs.

\section{REFERENCES}

1. De Morais Cordeiro, Carlos, Hrishikesh Gossain, and Dbarma P. Agrawal, "Multicast over wireless mobile ad hoc networks: present and future directions," IEEE network 17, no. 1, 2003, pp. 52-59.

2. Lavanya, P., V. Siva Kumar Reddy, and A. Mallikarjuna Prasad, "Research and survey on multicast routing protocols for MANETs," In 2017 Second International Conference on Electrical, Computer and Communication Technologies (ICECCT), IEEE, 2017, pp. 1-4.

3. Royer Elizabeth. M, and Charles E. Perkins, "Multicast operation of the ad-hoc on-demand distance vector routing protocol," in Proceedings of the 5th annual ACM/IEEE Int. Conf. on Mobile computing and Networking, ACM, 1999, pp.207-218.

4. Lavanya, P., V. Siva Kumar Reddy. V, and Mallikarjuna Prasad. A, "Exploring QoS multicast routing protocols for mobile ad hoc networks," in Proceedings of the 5th Int. Conf. on Computing, Communication and Sensor Network, 2016, pp.170-174.

5. Charles E. Perkins, and Elizabeth. M. Belding-Royer, "Quality of service for ad hoc on-demand distance vector routing, " Mobile Ad hoc Networking Working Group, Internet Draft, 14 Nov 2001.

6. For Network Simulator 2, http://www.isi.edu/nsnam/ns/ 
Multimedia Transmission through Mobile Ad hoc Networks using Multicasting

\section{AUTHORS PROFILE}

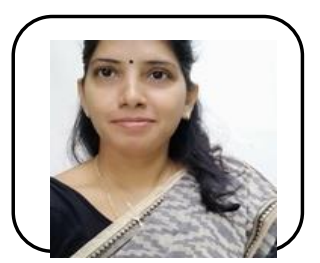

Lavanya. Poluboyina, completed B.Tech. in ECE from JNTU, Hyderabad, in 2004 and done M.Tech. in DSCE in the year of 2009. Has teaching experience of more than 15 years and presently working as Assistant Professor in the department of ECE, SNIST, Hyderabad. Areas of interest are Advanced Data Communications, Wireless Communications and Networks, Routing in Wireless Networks, Wireless Ad hoc Networks, and Mobile Ad hoc Networks. Member of IEEE and IEEE Communications Society.

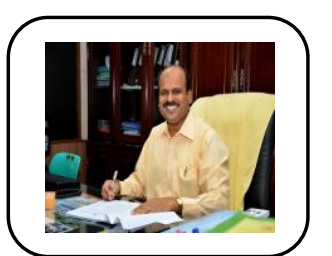

Sivakumar Reddy. V, Alumni of IIT Kharagpur, obtained Ph.D. in the area of Multi-media Signal Processing and Communication Protocols in 2008. Received M.Tech. degree in DS from JNTU College of Engineering, Hyderabad and B.Tech. from SVU, Andhra Pradesh. Has an experience of more than 19 years in teaching and industry put together and currently working as Principal, MRCET, Hyderabad. Published more than 45 research papers in the National and International Conferences and Journals. Areas of interest include Architecture for Signal Processing and Image/Video Coding and Video Communications, Computer Networks, TCP/IP Networks and Protocols, and Multimedia System Design. Fellow of IETE, Life Member of ISTE, and Member of IEEE.

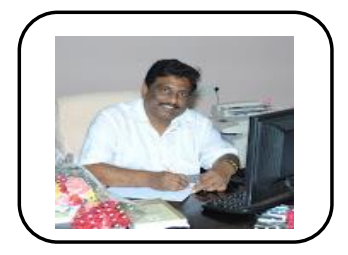

Mallikarjuna Prasad. A, received Ph.D. in ECE from JNTUH, Hyderabad in 2009. Completed M.E. from AU, Andhra Pradesh in 1992 and in 1988 done B.Tech. from Acharya Nagarjuna University, Andhra Pradesh. Has teaching and industry experience of more than 29 years together and presently working as Professor in the department of ECE, JNTUK, Kakinada. Have more than 50 research publications in the National and International Conferences and Journals. Research interests include Bio Medical Signal/Image Processing, Wireless Communications, Antennas, VLSI, and Electronic Instrumentation. 